\title{
CARACTERIZACIÓN ANÁTOMO-CLÍNICA DE LA FÍSTULA FISURARIA*
}

\author{
Drs. Cristóbal Suazo L. ${ }^{1}$, Guillermo Bannura C. ${ }^{1}$, Alejandro Barrera E. ${ }^{1}$, \\ Carlos Melo L. ${ }^{1}$, Felipe Illanes F. ${ }^{1}$, Cristián Gallardo V. ${ }^{1}$ \\ 1 Servicio y Departamento de Cirugía, Hospital Clínico San Borja Arriarán, Campus Centro, \\ Facultad de Medicina, Universidad de Chile. \\ Santiago, Chile.
}

\begin{abstract}
\section{Clinical and anatomical features of fissure-associated anal fistulae}

Background: Fissure-associated anal fistulae are not recognized in Parks classification and are probable underdiagnosed. Aim: To characterize these types of fistulae and describe their surgical management and long term results. Material and Methods: Review of medical records of a proctology surgical team. Identified patients were contacted by telephone to know their long term fate. Results: Twenty patients aged 29 to 59 years (70\% males), with fissure-associated anal fistulae were operated between 1998 and 2011 . These corresponded to $4 \%$ of all ano-rectal fistulae operated in the period. A fistulectomy was performed in $65 \%$ of patients and a fistulotomy in the rest. In $50 \%$ of patients, internal lateral sphicterotomy was also performed. Follow-up information was obtained in $80 \%$ of patients, 71 months (range 7-169) after surgery. No relapses or reoperations were recorded. Two female patients referred occasional gas incontinence, not affecting their quality of life. Conclusions: Considering the trajectory of fissure-associated anal fistulae, they do not affect the sphincter. The usual surgical treatment has a good long term prognosis, except in patients with active fissures, sphincter hypertonia or stenosis.
\end{abstract}

Key words: Anal fistula, anal fissure, anal sphincter.

\section{Resumen}

La fístula fisuraria (FF) es una fístula subcutánea no reconocida en la clasificación de Parks, probablemente existe un sub-diagnóstico de esta entidad. Objetivo: Caracterizar esta entidad desde el punto de vista anátomo-clínico, describiendo el manejo quirúrgico efectuado y sus resultados a largo plazo. Material y Método: Revisión retrospectiva de la base de datos de cirugía proctológica de nuestro hospital entre los años 1998-2011, con encuesta telefónica para conocer los resultados funcionales alejados. Resultados: Se intervinieron $20 \mathrm{FF}$, correspondientes al 3,9\% del total de fístulas anorrectales operadas en el período (70\%

*Recibido el 2 de octubre de 2013 y aceptado para publicación el 19 de diciembre de 2013.

Los autores no refieren conflictos de interés.

Correspondencia: Dr. Cristóbal Suazo L.

cristobalsuazolopez@gmail.com 
hombres), con una edad promedio de 47 años (extremos 29-59). Se realizó una fistulectomía en 65\% de los casos y una fistulotomía en el resto. Se asoció esfinterotomía lateral interna (ELI) en la mitad de los casos. Se completó seguimiento en $80 \%$ de los pacientes, con un promedio de 71 meses (extremos 7-169), sin recidivas de la fístula ni reoperaciones. Dos pacientes de sexo femenino con ELI refieren incontinencia ocasional a gases, que no afecta su calidad de vida. Conclusión: Dado el trayecto de la FF, la fistulectomía no afecta el aparato esfinteriano y tiene un buen pronóstico a largo plazo con el tratamiento quirúrgico habitual. No es necesario adicionar una ELI, salvo en pacientes con fisura activa y/o marcada hipertonía esfinteriana y/o estenosis moderada.

Palabras clave: Fisura anal, fístula fisuraria, fístula anorrectal, cirugía.

\section{Introducción}

Sir Alan Parks clasifica en 1976 las fístulas anorrectales de origen criptoglandular de acuerdo a la relación del trayecto fistuloso con el aparato esfinteriano, lo que aporta información anatómica relevante y orienta el manejo quirúrgico ${ }^{1}$. Esta clasificación no considera las fístulas de trayecto superficial, subcutáneas y/o submucosas, son habitualmente de trayecto corto y no tienen relación con el aparato esfinteriano. La literatura reconoce como mecanismos posibles de este tipo de fístulas subcutáneas la trombosis hemorroidal ulcerada, complicaciones de la cirugía formal de hemorroides en que se forman pliegues exuberantes que se cierran, abscesos luego de la inyección de toxina botulínica, el trayecto formado luego de una trombectomía anal y las fístulas originadas en el lecho de una fisura anal crónica. La fístula fisuraria (FF) se define como una fístula superficial que se origina en el lecho de una fisura anal profunda complicada con un absceso fisurario que drena hacia la piel perianal cercana al margen anal mediante un trayecto subcutáneo. Probablemente existe un sub-diagnóstico de esta entidad por falta de reconocimiento y muchas veces es clasificada como una fístula interesfinteriana simple. Es importante reconocer este tipo de fístula cuyo orificio fistuloso interno está por debajo de la línea pectínea por las implicancias terapéuticas ${ }^{2}$. En la literatura es mencionada como un tipo de fístula superficial en el contexto de estudios generales sobre las fístulas anorrectales ${ }^{3-7}$. El objetivo de este reporte es caracterizar a la FF desde el punto de vista anátomo-clínico, dar a conocer el manejo quirúrgico efectuado y los resultados funcionales a largo plazo.

\section{Pacientes y Método}

Se trata de una revisión retrospectiva de la base de datos prospectiva de cirugía proctológica de nuestro hospital entre los años 1998 y 2011. Se seleccionó a todos los pacientes con diagnóstico de fístula anorrectal operados en el período. En base a la información de la ficha clínica y del protocolo ope- ratorio se identificó a los pacientes con diagnóstico etiológico de FF. Se incluyen pacientes cuya fístula presenta las siguientes características: 1) Orificio fistuloso interno (OFI) por debajo de la línea pectínea; 2) Trayecto fistuloso subcutáneo (por dentro de la cara interna del esfínter interno), y 3 ) Orificio fistuloso externo (OFE) cercano al margen anal o en la base del plicoma centinela de la fisura anal crónica ${ }^{2,8}$ (Figura 1). La fisura anal puede presentarse total o parcialmente epitelizada. Se excluyeron del grupo de estudio los pacientes con otros tipos de fístulas subcutáneas, fístulas asociadas a condilomas, enfermedad de Crohn o recesos secundarios a cirugía proctológica. Los datos demográficos y la información clínica fueron obtenidos de la base de datos y complementados con la información registrada en la ficha clínica cuando fue necesario. El seguimiento de los pacientes se hizo mediante una encuesta telefónica con énfasis en los resultados funcionales a mediano y largo plazo.

\section{Resultados}

En el período de 14 años señalado se intervinieron 512 pacientes por una fístula anorrectal de diversa etiología, de las cuales $20(3,9 \%)$ corresponden a una $\mathrm{FF}$, el $70 \%$ del sexo masculino, con una edad promedio de 47 años (extremos 29-59 años). No hubo fístulas dobles o fístulas con más de un OFE.

En todos los pacientes existía el antecedente de una fisura anal crónica (proctalgia defecatoria con proctorragia escasa en forma intermitente) tratada en forma médica durante 3 meses hasta 5 años. El motivo actual de consulta es la aparición de una secreción anal intermitente con o sin dolor defecatorio asociado. Entre los hallazgos quirúrgicos destaca la presencia de una fisura parcialmente epitelizada en 8 casos y cicatrizada en 12, con papilitis hipertrófica y plicoma centinela en la mitad de ellos.

Se realizó fistulectomía en el $65 \%$ de los casos $(\mathrm{n}=13)$ y fistulotomía en los restantes $(\mathrm{n}=7)$ (Figura 2). Se asoció esfinterotomía lateral interna (ELI) en la mitad de los casos (6 fistulectomías y 4 fistulotomías). Se completó seguimiento en $80 \%$ 


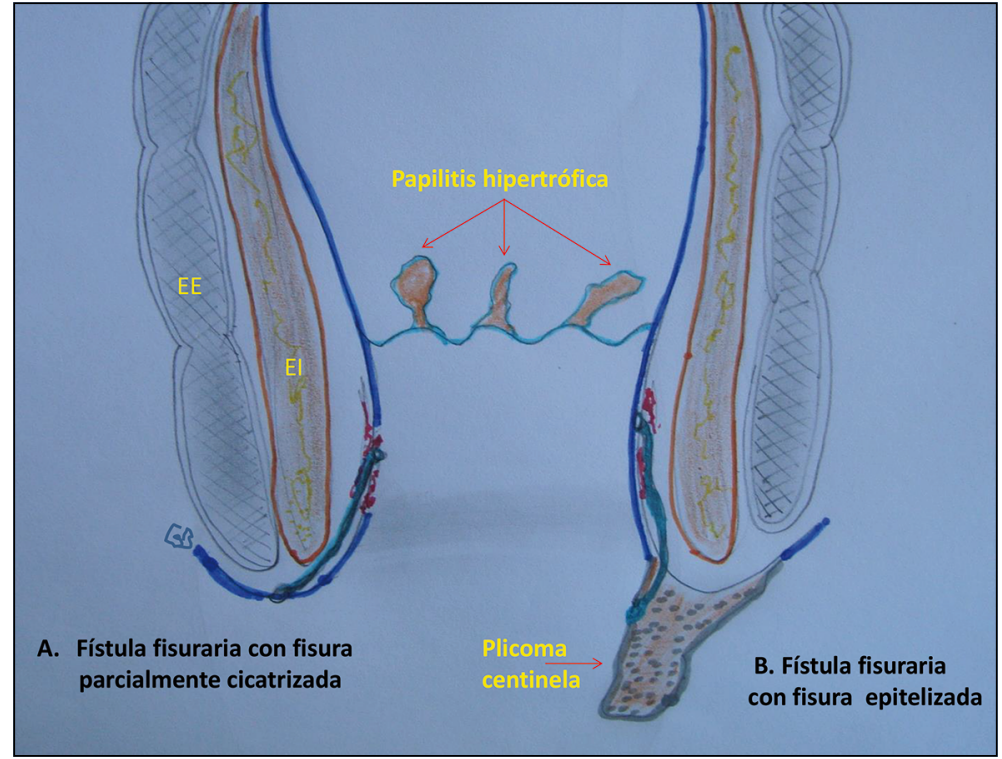

Figura 1. Dibujo esquemático de la FF. A: FF con úlcera activa (parcialmente cicatrizada). B: FF con fisura epitelizada. EE: esfínter externo; EI: esfínter interno.

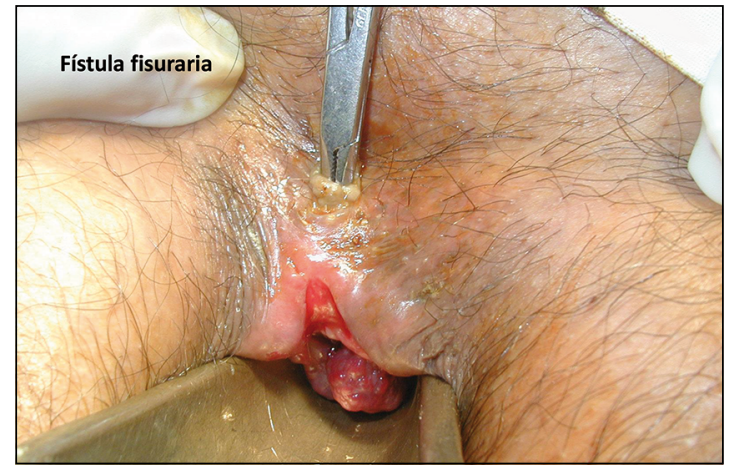

Figura 2. FF con trayecto subcutáneo identificado. Úlcera activa (parcialmente cictrizada).

de los pacientes (16), con un promedio de 71 meses (extremos 7-169), sin recidivas de la fístula ni reoperaciones. Dos pacientes de sexo femenino con ELI refieren incontinencia ocasional a gases, que no afecta su calidad de vida.

\section{Discusión}

Aunque las fístulas subcutáneas están brevemente mencionadas en los textos clásicos (Goligher, Corman), no hemos encontrado un estudio específico dedicado a este tipo de fístulas superficiales. La caracterización anátomo-clínica de la FF encontrada en este estudio confirma que se trata de una entidad distinta de las fístulas anorrectales de origen cripto- glandular descritas por Parks. La incidencia encontrada en esta revisión es concordante con lo descrito en la literatura que fluctúa entre 3 y $5 \%$, aunque es muy probable que un porcentaje de los casos no sea adecuadamente reconocido. De hecho, en nuestra revisión hubo casos que fueron identificados por la descripción y el esquema (dibujo) del protocolo operatorio, aunque no estaban nombrados como FF en el diagnóstico postoperatorio. Además, hemos comprobado en forma subjetiva, un aumento en el diagnóstico de esta entidad en la medida que existe una mayor difusión de sus elementos característicos entre los miembros del equipo tratante.

La sintomatología de la FF se imbrica con los síntomas de la fisura anal crónica, de modo que es difícil establecer un límite claro entre la patología de base y la complicación. Aunque el diagnóstico puede sospecharse durante el examen proctológico en la consulta, la confirmación del trayecto subcutáneo se realiza habitualmente en el pabellón quirúrgico, junto a la papilitis hipertrófica y el plicoma centinela que se presenta sólo en la mitad de los casos. La proctalgia defecatoria actual se presentó básicamente en los pacientes con una fisura activa, es decir, parcialmente epitelizada.

Si bien la FF es una patología de baja frecuencia, es importante reconocerla, ya que su tratamiento quirúrgico es simple y la mayoría de los casos se puede resolver con una fistulectomía que no afecta al aparato esfinteriano y, por lo tanto, no se asocia a riesgo de incontinencia. Generalmente en este tipo de fístulas la morbilidad está dada por la asociación del tratamiento de la fisura, como ocurrió en las dos 
pacientes sometidas a una ELI que en el seguimiento a largo plazo refieren incontinencia ocasional a gases. Aunque es controversial, la ELI podría justificarse en los pacientes con una fisura activa y una marcada hipertonía esfinteriana, procedimiento que no debe comprometer más allá del $40 \%$ de la longitud del esfínter ${ }^{9}$, efectuándola en un cuadrante lateral. Un argumento en contra de realizar una ELI es el hecho de que en los pacientes en que sólo se realizó el tratamiento quirúrgico de la fístula no hubo recidiva de la fisura ni de la fístula. La decisión entre realizar fistulotomía o fistulectomía fue exclusivamente discrecional y aparentemente no habría diferencias en los resultados funcionales a largo plazo.

En conclusión y de acuerdo con los hallazgos de esta revisión, se puede definir anatómicamente una fístula fisuraria por tener un trayecto superficial o subcutáneo corto entre el lecho de una fisura crónica, parcial o completamente cicatrizada, y un orificio fistuloso externo a 1-2 cm del margen anal o en la base del plicoma centinela de la fisura. Por definición la FF no compromete el esfínter interno, la fistulectomía no afecta el aparato esfinteriano y tiene un buen pronóstico a largo plazo con el tratamiento quirúrgico habitual. La necesidad de adicionar un tratamiento quirúrgico específico para el manejo de la fisura anal podría estar justificada por la presencia de una fisura activa con marcada hipertonía esfinteriana y/o por estenosis moderada. No es necesario asociar una ELI a todos los pacientes portadores de una FF, especialmente mujeres, evitando de esta forma el riesgo de incontinencia asociado.

\section{Referencias}

1. Parks AG, Gordon PH, Hardcastle JD. Classification of fistula-in-ano. Br J Surg. 1976;63:1-12.

2. Bannura G, Ricaurte F, Scandar P. Resultados del tratamiento quirúrgico de la fístula anorrectal: análisis de 70 pacientes. Rev Chil Cir. 1999;51:615-22.

3. Abcarian H. Surgical correction of chronic anal fissure: results of lateral internal sphincterotomy vs fissurectomy for anal fissure. Dis Colon Rectum. 1980;23:31-6.

4. Casal E, San Ildefonso AD, Sánchez J, Facal C, Pampin J. Fístula anal de origen criptoglandular. Opciones terapéuticas. Cir Esp. 2005;78 (Supl 3):28-33.

5. Barbosa RK, Manson R, Fourcans S, Foniol MG, Amarillo HA, Amarillo HR. Tratamiento quirúrgico de la fístula anal, análisis de nuestra experiencia. Rev. Argent Cir. 2009;96:102-9.

6. Sainio P, Husa A. Fistula-in-ano. Clinical features and long-term results of surgery in 199 adults. Acta Chir Scand 1985;151:169-76.

7. Bussen D, Sailer M, Wening S, Fuchs KH, Thiede A. Usefulness of anal endosonography in the assessment of fistula-in-ano. Zentralbl Chir. 2004;129:404-7.

8. Bannura G, Cumsille MA, Ricaurte F, Tepper P, Pfeiffer B. Resultados alejados del tratamiento de la fístula anorrectal: análisis de la recidiva y la continencia en 159 pacientes. Rev Chil Cir. 2002;54:189-95.

9. Murad-Regadas SM, Fernandes GO, Regadas FS, Rodrigues LV, Pereira J de J, Regadas Filho FS, et al. How much of the internal sphincter may be divided during lateral sphincterotomy for chronic anal fissure in women? Morphologic and functional evaluation after sphincterotomy. Dis Colon Rectum 2013;56:645-51. 